\title{
Des Autrices Transatlantiques?
}

\section{Yves Clavaron}

\section{Université de Lyon, UJM-Saint-Etienne (CELEC, EA 3069)}

Résumé: L'article se propose d'examiner dans quelle mesure on peut parler d'autrice transatlantique à partir de certaines œuvres de Maryse Condé, Chimamanda Ngozi Adichie et Taiye Selasi. Les études transatlantiques, que l'on peut situer entre les études postcoloniales et les études de la globalisation, proposent de relire les phénomènes se déroulant sur les continents bordés par l'Atlantique - Europe, Afrique, Amériques - en prenant en compte les échanges pluridirectionnels à l'œuvre ainsi que l'intégration progressive au sein de cet espace de dynamiques occidentales et extra-occidentales dans une histoire littéraire connectée. Il s'agit de montrer comment certaines autrices construisent une œuvre littéraire transatlantique en mettant en fiction une problématique identitaire, géopolitique et culturelle, notamment une construction socio-culturelle des identités féminines, selon une poétique transnationale.

Mots-clés: autrice, transatlantique, subalternisme, féminisme, poétique, transnational

Abstract: The purpose of this paper is to examine to what extent would it make sense to speak about transatlantic authoress considering some works of Maryse Condé, Chimamanda Ngozi Adichie and Taiye Selasi. Transatlantic studies, which could be located between postcolonial and globalization studies, aim at reviewing processes occurring on the continents surrounded by the Atlantic Ocean (Europe, Africa and America) taking account of the multi-pronged exchanges at work as well of the gradual integration into this space of western and non-western dynamics within the framework of a connected literary history. This entails showing how some authoresses create a transatlantic literary work by putting into fiction an identity, geopolitical and cultural problem, mainly a sociocultural building of female identities, according to a transnational poetics.

Keywords: authoress, transatlantic, subalternism, feminism, poetics, transnational 
Parler d'“autrice" et donc imposer une féminisation du terme "auteur" apparaît comme un casus belli au xvii siècle, notamment après la décision de l'Académie française prononcée dans le Dictionnaire de 1762: “En parlant d'une femme qui aura composé un livre, on dit qu'Elle est l'Auteur d'un tel livre, d'un tel ouvrage" (Viennot 2017: 54). Pourtant le doublet "auteur/autrice" ne dérangeait personne au xvie siècle, pas plus que ne dérange aujourd'hui le doublet acteur/actrice. ${ }^{1}$ L'expression "Femme autrice" est un pléonasme qui souligne le caractère inconcevable de l'auctorialité au féminin (idem: 53).

Pour en revenir à la fonction plus générale de l'auteur, il apparaît comme indispensable pour la littérature. L'auteur ou l'auctor, celui qui est à l'origine de quelque chose, représente l'autorité, l'auctoritas. Le terme implique une responsabilité, juridique ou intellectuelle. L'autorité de l'écrivain est liée à la valeur de ce qu'il produit et le "grand" auteur appartient au canon littéraire. L'auteur est celui dont l'œuvre se situe parmi les "monuments" et non les "documents" pour reprendre l'opposition développée par Michel Foucault: "De nos jours, l'histoire c'est ce qui transforme les documents en monuments" (1969a: 15). Il ne faut pas, en effet, confondre l'auteur et le simple rédacteur.

L'auteur est une notion qui a émergé lentement, avant de se fixer entre les Lumières et le Romantisme. La notion d'auteur n'existait ni dans l'Antiquité ni au Moyen Âge, où l'auteur représentait une parole anonyme et où le véritable auteur était Dieu. C'est au xviie siècle que naît la figure de l'auteur comme le montre Alain Viala (1985), quand précisément le féminin se trouve exclu. L'auteur gagne progressivement une autonomie, un pouvoir propre qui conduit à son "sacre" dans la seconde moitié du xviiie siècle (Bénichou 1973). La figure de l'auteur, homme de lettres ou philosophe, prend la place d'un pouvoir religieux, jugé défaillant, et devient législateur du genre humain au nom d'une foi humaniste. La période romantique confirme la sacralité de l'auteur, véritable mage qui se veut guide politique du peuple à l'instar de Lord Byron ou de Victor Hugo. Au xix e siècle, l'auteur se trouve pris entre tentation du retrait - "la disparition élocutoire du poète" de Mallarmé - et l'engagement - le "J'accuse" de Zola, même si, par ailleurs "l'industrialisation de la littérature et la montée en puissance des grands éditeurs" menacent son statut symbolique (Compagnon). La position de l'auteur a connu des variations importantes au $x^{e}$ siècle: 
après avoir presque disparu sous l'influence de la critique formaliste et structuraliste qui a privilégié la notion d'écriture et de texte - "Qu'importe qui parle?" demandait Foucault (1969b), l'auteur fait un retour en force avec la postmodernité même si les nouveaux médias numériques remettent en cause son statut juridique. La disparition de l'auteur était un leurre, "un moyen paradoxal de sa figuration: son absence est un mode négatif de sa présence" (Brunn / Martin 2019: s.p.).

Nous voudrions articuler la figure de l'auteur ou plutôt de l'autrice en l'occurrence aux études transatlantiques, que l'on peut situer entre les études postcoloniales - les situations historiques examinées sont des produits de l'expansion coloniale de l'Europe - et les études de la globalisation sur les flux et les échanges planétaires, notamment la littérature mondiale. La perspective des études atlantiques propose de relire les phénomènes se déroulant sur les continents bordés par l'Atlantique - Europe, Afrique, Amériques - en prenant en compte les échanges pluridirectionnels à l'œuvre ainsi que l'intégration progressive au sein de cet espace de dynamiques occidentales et extraoccidentales dans une histoire littéraire connectée.

Ainsi, il s'agit pour nous de montrer comment certaines autrices construisent une œuvre littéraire transatlantique en mettant en fiction une problématique identitaire, géopolitique et culturelle, notamment une construction socio-culturelle des identités féminines, mais sans exclusive. Pour cela, nous nous appuierons sur les œuvres de trois autrices noires emblématiques, une pionnière des littératures dites postcoloniales en français, Maryse Condé, consacrée en 2018 par le prix Nobel alternatif pour une œuvre qui s'étend sur plus de quarante ans, et deux écrivaines plus jeunes, Taiye Selasi et Chimamanda Ngozi Adichie, ressortissantes du monde anglophone et autrices de romans qui ont eu un énorme succès en 2013, respectivement Ghana Must Go et Americanah. Leur langue d'expression est européenne: le français et l'anglais, retravaillés en fonction d'un contexte socioculturel nouveau, l'espace atlantique qui intègre les écritures des Suds. Pour éviter de tomber dans une essentialisation du féminin, on invoquera l'“essentialisme stratégique" un temps soutenu par Gayatri Spivak (2011: 78), ${ }^{2}$ qui consiste à entrer dans le point de vue du dominant afin de déconstruire les systèmes hégémoniques, souvent mis en 
place par des hommes, bref une forme de revanche postcoloniale dans une "black Atlantic" au féminin (Gilroy 2003). ${ }^{3}$

\section{L'autrice subalterne peut parler (et écrire)}

L'autrice transatlantique est a priori éloignée de la figure de l'auctor en majesté, du "grand auteur", celui qui garantit le sens et produit un "monument". Plus encore que son homologue masculin, une autrice transatlantique est une voix issue d'une minorité et d'une périphérie par rapport à un centre européen ou états-unien. Conscience agissante, elle se situe souvent par rapport aux courants féministes, qu'ils soient occidentaux ou non. La femme subalterne a subi une oppression plus forte que la femme bourgeoise blanche, ce qui fait écarter par certaines féministes afro-américaines comme bell hooks le concept de “sororité": "L’appel à la sororité a en effet souvent été perçu comme une manœuvre manipulatrice et opportuniste des bourgeoises blanches, un vernis rhétorique servant à masquer l'exploitation et l'oppression perpétuées par des femmes sur d'autres femmes" (hooks 1986: 7).

\section{Voix minoritaires et périphériques transatlantiques}

L'autrice transatlantique fait écho à la figure de la subalterne décrite par Gayatri Spivak, telle la femme indienne qui, présence muette et symbole absolu de l'aliénation, est dépourvue d'histoire et souffre d'une invisibilité plus grande encore que son compatriote homme. Doublement colonisée (Holst-Petersen / Rutherford 1985), la femme "genrée" constitue l'essence de la subalternité, soumise à une violence épistémique:

Entre le patriarcat et l'impérialisme, la constitution du sujet et la formation de l'objet, la figure de la femme disparaît, non dans un néant virginal, mais dans un violent va-et-vient qui correspond à la figuration déplacée de la "femme du Tiers-Monde", prise entre tradition et modernisation. (Spivak 2009b: 98) 
Gayatri Spivak insiste sur la position de la femme colonisée, subalterne au carré dans une société patriarcale et (post)coloniale, exclue de la sphère du discours et de la représentation. Elle se situe hors des systèmes, même si elle finit par être intégrée à certains réseaux ensuite, notamment numériques. En cela, Gayatri Spivak se démarque d'une vision marxiste, qui fait du subalterne un sujet nécessairement conscient d'un point de vue politique. La figure de la subalterne serait par excellence celle qui ne peut être autrice, étant dépourvue de toute forme d'autorité et de légitimité. Pour autant, les études subalternistes tendent à faire des subalternes une sorte d'auteur collectif, conscient de soi et finalement acteur politique pour peu que l'on se libère de l'optique et de l'archive coloniales. Quant à Gayatri Spivak, elle distingue les statuts de lecteur/lectrice et d'auteur/autrice: "Ceux qui lisent ou écrivent de la littérature peuvent aussi peu prétendre à un statut subalterne que ceux qui lisent ou écrivent de l'histoire" (2009a: 426). Une autrice - au même titre que la professeure et la lectrice - ne saurait donc être une subalterne car elle occupe une position de sujet, notamment de l'écriture.

Maryse Condé, Taiye Selasi et Chimamanda Ngozi Adichie appartiennent à des minorités à plus d'un titre, en tant que femmes, noires et écrivaines. Française, Maryse Condé est ressortissante des Caraïbes (la Guadeloupe), mais son parcours transatlantique se construit entre l'Afrique, qui a constitué une première inspiration de son œuvre culminant avec Ségou, et l'Amérique, notamment les États-Unis, où elle s'est installée pendant une période de sa vie et qui a constitué la matière d'une autre partie de son œuvre, inaugurée par Moi, Tituba Sorcière... En toile de fond, se trouve la métropole puisque la Guadeloupe est un département français et que la plupart des instances de légitimation francophones se trouvent à Paris.

Britannique, Taiye Selasi est d'origine africaine, née à Londres d'une mère d'origine nigériane et d'un père ghanéen, tous les deux médecins, et a grandi dans le Massachussetts. Elle circule dans le monde entier, mais est désormais installée à Rome, qui peut apparaître comme un espace neutre, hors de l'univers anglophone. Comme elle l'explique dans son essai “Bye-bye Babar (Or: What is an Afropolitan?)” (Selasi 2014a : 322-326), Taiye Selasi se considère comme une "afropolitaine", appartenant à la génération émergente d'Africains 
qui sont les produits des cultures urbaines de l'Occident où leurs parents ont émigré dans les années 1970-80 (Alix 2017). En effet, l’afropolitanisme désigne la dimension cosmopolite de l'Afrique, à la fois par les déplacements intra-africains et par les relations intercontinentales induits par la mondialisation, tout en valorisant l'image de l'Africain intégré à une élite transculturelle. Pour sa part, l'afropéanisme (Miano 2012) privilégie, dans un entre-deux entre l'Afrique et l'Europe, les identités transfrontalières au sein de la diaspora noire, les personnes d'ascendance subsaharienne ou caribéenne et de culture européenne. Les deux notions disent le refus de la racine unique et prennent acte de la présence de l'ailleurs dans l'ici et de l'ici dans l'ailleurs.

Chimamanda Ngozi Adichie refuse, quant à elle, l'étiquette d'"afropolitain": "Pourquoi imposer aux Africains un mot particulier, lorsqu'ils ne font que ce que tout le monde fait: ils voyagent, ils sont modernes, ils inventent?" (Adichie apud Simon 2015: s.p.), s'insurge-t-elle. Il faut dire que l'afropolitanisme transcende les questions raciales qui demeurent centrales dans son roman Americanah, qui se conclut en outre par une réinstallation au pays natal, mettant fin à la mobilité. Nigériane, Chimamanda Ngozi Adichie écrit depuis l'Afrique même si, vivant entre le Maryland et Lagos, elle est une écrivaine migrante, passant sans cesse d'un côté de l'Atlantique à l'autre. Elle trouve à s'incarner dans son roman Americanah qui traite de la condition de l'émigré africain aux États-Unis et moque par son titre les Nigérians trop américanisés. Chimamanda Ngozi Adichie évoque le Nigerpolitan Club à Lagos, qui réunit les Nigérians de retour au pays, communauté interculturelle passablement snob et insatisfaite, qui ne cesse de comparer les deux côtés de l'Atlantique (Adichie 2014: 407; Adichie 2016: 585). À travers les années américaines de l'héroïne d'Americanah, Ifemelu, qui voient l'élection de Barack Obama à la présidence des États-Unis - et celles, londoniennes, d'Obinze, Chimamanda Ngozi Adichie pose la question du racisme et du sexisme dans les grandes métropoles occidentales sur le mode de l'humour. 


\section{La question du féminisme}

Le discours des autrices s'articule face à un ensemble constitué de valeurs dominantes et centrales, associées à l'Europe ou à l'Occident en général. Ces valeurs sont celles du capitalisme, de la bourgeoisie, de la masculinité ou de la rationalité. Leurs œuvres s'enracinent dans de multiples formes de contestations des centralités, spatiales l'eurocentrisme -, axiologiques - la prédominance des valeurs de la bourgeoisie blanche -, historiques - le récit imposé par l’historiographie occidentale - ou génériques l'hégémonie masculine. Elles remettent en cause le patriarcat universel, en dévoilant le visage multiforme de la domination masculine.

Comme le montre Chloé Chaudet (2017) à partir d'un corpus d'écrivaines postcoloniales, les textes se caractérisent par une mise en valeur de l'intersectionnalité des discriminations. Le concept d'intersectionnalité est apparu au sein des études féministes dans un article de Kimberlé Crenshaw (1989): il renvoie à une théorie transdisciplinaire visant à appréhender la complexité des identités et des inégalités sociales à travers la prise en compte des intersections entre race, classe et genre. Kimberlé Crenshaw insiste sur la multiplicité des systèmes d'oppression qui se coalisent et se co-déterminent avec pour conséquence la marginalisation et la subalternisation des femmes noires. C'est aussi l’optique du "Black Feminism" illustré par Angela Davis et Alice Walker en tant que "courant de pensée qui, au sein du féminisme, a défini la domination de genre sans jamais l'isoler des autres rapports de pouvoir, à commencer par le racisme ou le rapport de classe" (Dorlin 2008: 21).

Dans Americanah, l'autrice met également au jour la diversité des dominations masculines, liées à des contextes géographiques, historiques et culturels spécifiques. L'héroïne en fait l'expérience à travers ses diverses relations masculines, souvent associées à une expérience raciale: Curt, le Blanc "sexy" et riche, dans la maison duquel "le seul autre Noir était un serveur en uniforme" (Adichie 2016: 297) ("The only other black person was a stiffy dressed waiter" (Adichie 2014: 198)), Blaine, l'universitaire africain-américain qui se trouve irrité par la proximité d'Ifemelu avec Boubacar, le Sénégalais, "quelque chose d'essentiellement africain dont il se sentait exclu" (Adichie 2016: 498) ("something primally 
African from which he felt excluded" (Adichie 2014: 340)), et Obinze, l'amour de jeunesse du Nigeria vers lequel elle finira par retourner. Son féminisme est jugé parfois un peu aseptisé ou trop conciliant comme en témoigne une conférence prononcée en 2012: “J'ai décidé d'être une Féministe Africaine Heureuse qui ne déteste pas les hommes, qui aime mettre du brillant à lèvres et des talons hauts pour son plaisir, non pour séduire les hommes" (Adichie 2018: 20). Elle définit comme féministe, toute personne homme ou femme qui considère que "la question du genre telle qu'elle existe aujourd'hui pose problème" (idem: 50) et que "nous devons faire mieux" (ibidem).

Le premier texte publié par Taiye Selasi dans la revue Granta en 2011 s'intitule "The Sex Lives of African Girls" et commence par une phrase qui complète le titre: "Begin, inevitably, with Uncle” (Selasi 2011: s.p.) (“Commence, inévitablement, avec l’Oncle”). Or, Edem, âgée de onze ans, a été emmenée encore enfant par son oncle pour vivre auprès de lui à Accra. Dans Ghana Must Go, l'abus sexuel ne concerne pas que les filles. Par ailleurs, le roman met en scène un personnage féminin traditionnel, Ama, la seconde et jeune épouse de Kweku, qui n'est pas sans personnalité, mais dont la vie allie consentement - elle dit toujours "oui" - et contentement car elle possède "la faculté de rester heureuse" (Selasi 2014b: 68) ("the capacity to stay happy" (Selasi 2014a: 50)). Son acquiescement à l'ordre social patriarcal ne la prive pas de sa liberté car cette femme-enfant semble comme ravie au monde vis-à-vis duquel elle conserve une sereine indépendance. Son dernier geste est de rapporter à la première épouse, Folá, les pantoufles cabossées que Kweku avait perdues au moment de mourir, emballées dans un sac portant l'inscription éponyme "Ghana Must Go". Le roman interroge les relations hommes/femmes notamment à travers la fratrie Sai, mais sans acrimonie. Taiye Selasi pense avoir hérité le féminisme de sa mère, mais qui ressort dans son écriture comme filtré et apaisé:

I suspect [my mother's] feminism expresses itself in my work: my female characters speak their minds, their truths, however quietly. This always just sort of happens: the women appear on the page with a wisdom of their own. I'm not aware of writing with a political agenda, however obvious it is that I've inherited one. I write to write. (Selasi apud Akfbar 2011: s.p) 
[Je soupçonne le féminisme [de ma mère] de s'exprimer dans mon œuvre: mes personnages féminins disent ce qu'ils pensent, en toute franchise, mais sereinement. C'est toujours ainsi que cela se passe: les femmes apparaissent sur la page avec une sagesse à elles. Je n'ai pas le sentiment d'écrire avec un programme politique, quoiqu'il soit évident que j'en ai hérité un. J'écris pour écrire.]

Taiye Selasi est intimement persuadée qu'une femme doit pouvoir s'exprimer tout à fait librement, sans se sentir infériorisée par le fait qu'elle est une femme (Gleeson 2013).

Maryse Condé revendique également une parole libre, indépendante de son statut de femme. Elle évoque des modèles féminins qui l’ont influencée (Brugier 2016), mais se défend d'être féministe:

J'ai été élevée par ma mère et ma grand-mère, deux caractères forts. Tout comme mes sœurs. Et comme les autres femmes que j'ai connues en Guadeloupe. Elles n'abdiquaient pas face à l'adversité. Je ne me considère pas comme une féministe. J'écris sur ce que je connais. (Condé 2000: 50)

Elle peut néanmoins passer pour féministe dans la mesure où elle formule sans ambages le discours du désir féminin. Elle fait de Tituba la femme qui dit et vit son désir et transgresse les tabous de la société coloniale et patriarcale portés à leur paroxysme par la mentalité puritaine. Tituba est stigmatisée par le village de Salem, qui en fait un bouc émissaire dans une société où le corporel et le sexuel sont marqués au fer écarlate d'une lettre "A" pour "adultery": “On ne lapide plus les femmes adultères. Je crois qu'elles portent sur la poitrine une lettre écarlate" (Condé 1986: 155). En prison, Tituba devient amie avec Hester, punie pour sa liberté amoureuse et toutes deux s'entretiennent longuement sur leurs besoins sexuels. Hester apparaît comme une féministe militante qui imagine une république des femmes, "une société gouvernée, administrée par les femmes. Nous donnerions notre nom à nos enfants, nous les élèverions seules..." (idem: 159-160). Elle reproche d'ailleurs à Tituba d'être aliénée par ses désirs sexuels: "Tu aimes trop l'amour, Tituba! Je ne ferai jamais de toi une féministe!” (idem: 160). Si l'entretien peut paraît décalé dans le contexte de l'Amérique puritaine du XVII e siècle, son contenu relève d'un féminisme radical qui n'est pas celui de Maryse Condé, d'où la dimension parodique qu'elle a pu alléguer dans des entretiens ultérieurs avec Françoise Pfaff (1993: 90). Les trois autrices se 
situent donc différemment par rapport au féminisme, sur un mode parodique chez Maryse Condé, conciliant pour Chimamanda Ngozi Adichie et tempéré chez Taiye Selasi grâce à l’héritage maternel, les trois excluant l'idéologie d'un féminisme radical.

\section{Poétiques transatlantiques}

Les autrices sont confrontées à un espace tricontinental durablement marqué par les pratiques de l'impérialisme européen et occidental en général. Il s'agit pour elles d'en faire un espace littéraire caractérisé par une poétique qui dépasse les frontières nationales des genres et des canons littéraires. L'étymologie du nom "auteur" est également associée aux verbes augere ("augmenter" et "garantir") et agere ("agir"). L'autrice exprime son agency (sa puissance d'agir) par l'élaboration d'une poétique qui vient augmenter la réalité et affirmer une vision du monde.

\section{Construction de l'espace littéraire transatlantique}

L'espace transatlantique, précolonial, colonial et postcolonial se trouve soumis à la création littéraire, souvent dans une perspective critique de miroir inversé par rapport à l'Europe. Pour Maryse Condé, il s'agit de repenser un monde nouveau ou un nouveau monde à partir d'une expérience historique tragique: la mémoire douloureuse de l'esclavage et de la traite. Paul Gilroy (1993) présente d'ailleurs le souvenir de la violence esclavagiste comme cadre essentiel à partir duquel s'élabore le fondement culturel de la diaspora noire. La romancière inscrit son roman Ségou dans l'héritage de douleurs et de transplantations forcées subies par les Africains, ainsi que l'expérience du cycle long de la colonisation et des indépendances avec son lot de transferts culturels et de situations d'acculturation. L'espace précolonial africain est soumis à la représentation littéraire dans Ségou, mais c'est l'espace colonial américain qui est représenté dans Moi, Tituba Sorcière... au temps de la domination britannique.

Maryse Condé avait réuni des matériaux ethnographiques sur les traditions orales

N. 940 - 06/ 2019 | 67-89 - ISSN 2183-2242 | http:/dx.doi.org/10.21747/21832242/litcomp40a3 
mandingues dans l'intention de rédiger une thèse d'État, mais elle préfère finalement en faire un roman en deux volumes: Ségou. Les Murailles de la terre (1984) et Ségou. La Terre en miettes (1985). C'est ainsi qu'elle déploie une grande fresque historique qui signifie la disparition d'une certaine Afrique représentée par le royaume bambara de Ségou. Les deux volumes évoquent des périodes distantes par rapport au présent de l'écrivaine: le premier, commençant à la fin du XVIIIe siècle, illustre l'islamisation souvent violente de l'Afrique subsaharienne, le second, débutant vers 1860, marque l'inexorable avancée de la colonisation européenne. Le voyage vers l'Afrique correspond à une recherche des racines car, aux Antilles, "il n'y a pas d'ancêtre fondateur, il n'y a que le bateau négrier" (Condé apud Chanda 2018: s.p.). Maryse Condé se tourne ensuite vers l'Amérique du nord, tout en restant fidèle au genre historique, avec son roman Moi, Tituba Sorcière... Noire de Salem (1986) qui inaugure son intérêt pour les États-Unis, pays qu'elle découvre alors. Le personnage éponyme a réellement existé et figure au nombre des accusées du célèbre procès en sorcellerie qui se déroula à Salem, bourg du Massachusetts, en 1692. Le roman repose donc sur un fait historique avéré par les archives, qu'il s'agira de lire "against the grain" ("à contre-fil") selon les préconisations de l'historien subalterniste Ranjit Guha car l'esclave noire se trouve quasiment invisible, largement ignorée par l'historiographie qui la relègue à un statut de "comparse sans intérêt” (Condé 1986: 183).

Les romans de Taiye Selasi et Chimamanda Ngozi Adichie se situent à l'époque contemporaine entre Afrique et Amérique, mais les autrices restent les héritières d'une histoire transatlantique. Ainsi, une grande partie du roman Ghana Must Go de Taiye Selasi se déroule au Ghana, où il s'ouvre avec le décès brutal par infarctus du patriarche de la famille, Kweku Sai. Cette mort est le point de départ d'un faisceau de récits qui structurent la narration en remontant jusqu'aux origines de la famille. Kweku Sai, le père, victime d'une injustice professionnelle liée à un racisme latent est renvoyé de l'hôpital de Boston où il exerce comme chirurgien. Humilié, il dissimule son sort jusqu'à ce que son fils découvre la réalité, ce qui entraîne sa fuite au Ghana et la dissémination de la famille (Chanda 2014). Le roman se construit par-dessus l'Atlantique dans une relation entre Amérique et Afrique, qui marque une distance avec l'insistance sur l'océan séparant Boston, les États-Unis, du Ghana, 
le fils du père mort, mais opère aussi la fusion d'un système antagoniste grâce à la seconde épouse de Kweku, Ama. Celle-ci ressemble à sa fille Taiwo et, de ce fait, réussit à concilier la modernité et la tradition par-dessus l'Atlantique, son "corps doux" constituant "un pont entre les deux mondes sur lequel il marche" (Selasi 2014b: 71), ("whose soft body is a bridge on which he walks between worlds" (Selasi 2014a: 52)). Dans Americanah, les circulations transatlantiques révèlent tout d'abord une forme d'identité; l'autrice a découvert la "race" aux États-Unis: "Moi-même je ne me sentais pas noire, je ne suis devenue noire qu'en arrivant en Amérique" (Adichie 2016: 428), ("I did not think of myself as black and I only became black when I came to America" (Adichie 2014 : 290)) et "En descendant de l'avion à Lagos, j'ai eu l'impression d'avoir cessé d'être noire" (Adichie 2016: 683), ("I feel like I got off the plane in Lagos and stopped being black" (Adichie 2014: 476)). Ce sentiment lui fait écrire sur son blog "Parfois en Amérique, la race est la classe" (Adichie 2016: 252), ("Sometimes in America, Race Is Class" (Adichie 2014: 166)). Le texte montre les constructions mythologiques qui s'élaborent de part et d'autre de l'Atlantique: les Nigérians, installés aux États-Unis, rentrent au pays pour lire leur brillante réussite américaine dans le regard de leurs compatriotes tandis qu'en Amérique, ils construisent de véritables chimères sur leur pays d'origine qu'ils défendent aveuglément sur internet alors même que leur identité se dissout dans un entre-deux, "entre ici et là-bas" (Adichie 2016: 178), "between here and there" (Adichie 2014: 117).

La construction d'un espace littéraire transatlantique se développe à partir de la thématique très postcoloniale du déplacement et de la dissémination: voyage, émigration, exil, diaspora, retour aux sources en Afrique. Le trope de la mobilité est associé à la précarité et à une angoisse fondatrice, mais ces problématiques ne sont pas exclusivement féminines. Les personnages de Taiye Selasi vont et viennent entre États-Unis, Ghana et Nigeria, ceux de Chimamanda Ngozi Adichie entre Nigeria, États-Unis et Grande-Bretagne, tandis que ceux de Maryse Condé sont soumis pour certains au "middle passage", qu'il s'agisse de Naba dans Ségou (1984: 81), embarqué pour les plantations du Brésil, ou d'Abena, la mère de Tituba, pour l'île de la Barbade dans Moi, Tituba Sorcière... Le texte transatlantique semble défini par une relation intime avec le contexte culturel et politique. 
Les romans de Maryse Condé, Taiye Selasi et Chimamanda Ngozi Adichie configurent, dans et par la fiction, une revendication identitaire, politique mais aussi poétique, qui implique le réseau intercontinental, tout en scrutant le transfert transocéanique, principalement entre Afrique et Amérique.

\section{Poétique transnationale}

L'usage de la langue, la pratique de l'intertextualité, le recours au canon ou son rejet constituent des indices du travail d'appropriation, de réappropriation ou de désappropriation à l'œuvre dans un roman "transatlantique". La pratique de la réécriture ou la reprise de modèles littéraires reflète l'autorité de l'auteur ou de l'autrice, son agency en matière d'écriture liée à sa force de décentrement. Maryse Condé, Taiye Selasi et Chimamanda Ngozi Adichie proposent un traitement particulier de la mémoire et des héritages européens, africains et américains, qui passent par la réinvention, la contestation ou la digestion des lettres métropolitaines européennes. Les trois autrices produisent des littératures qui adoptent des perspectives minoritaires ou diasporiques et ne peuvent se définir par un label national. Le caractère transnational de leur poétique s'inscrit au sein d'un cosmopolitisme que l'on peut appeler World Literature ou Littérature-monde et correspond à une esthétisation du fait diasporique dans un contexte d'intégration à la globalisation.

Réécrivant l'histoire de sorcières de Salem du point de vue d'une esclave noire, Maryse Condé fait référence à un hypotexte, non pas français mais américain, le roman The Scarlet Letter (1850) de Nathaniel Hawthorne, un des textes fondateurs du canon étatsunien. Elle procède à une réécriture d'un épisode important, l'emprisonnement d'Hester Prynne que Tituba, la comparse, rencontre en prison contre toute vraisemblance chronologique. La vision de la vieille femme la corde au cou sur une estrade à Boston (Condé 1986: 81) apparaît d'ailleurs comme une réécriture du chapitre deux de The Scarlet Letter, où Hester est mise au pilori sur la place du Marché à Boston. La dimension parodique consiste à déconstruire la figure héroïque romanesque centrale du roman en lui faisant porter d'autres valeurs, celles d'un féminisme de la révolte. Le roman de Maryse Condé 
commence comme celui de Nathaniel Hawthorne par un (para)texte préliminaire attestant l'authenticité du récit qui, en fait, contribue à donner un caractère légendaire au personnage féminin mis en scène.

Chimamanda Ngozi Adichie évoque, elle, le roman picaresque Huckleberry Finn de Mark Twain, mais l'héroïne relègue très vite le texte parmi les "âneries illisibles" (Adichie 2016: 107), ("unreadable nonsense" (Adichie 2014: 67)), même s'il est considéré comme un "livre sérieux" par Obinze, faute sans doute d'avoir bien compris les dialectes américains, différents de l'anglais du Nigeria. Chimamanda Ngozi Adichie joue sur les variations de l'énonciation: assumée en alternance par chacun des membres du couple séparé, Ifemelu et Obinze, la narration est entrecoupée de billets du blog qui mettent en scène la protagoniste en tant que Noire d'origine non-américaine et créent une nouvelle sociabilité avec la communauté visée. Significativement, le blog commence après la rupture d'Ifemelu avec Curt, son amant blanc, moment aigu de prise de conscience "raciale". Les billets du blog se démarquent du reste du texte par une différenciation typographique et participent d'une esthétique hybride où écriture fictionnelle et commentaire social interagissent continuellement. Grâce à son statut d'observatrice extérieure, Ifemelu s'attaque à une multiplicité de clichés sur la race et le genre. Peu réceptive au picaresque même si son roman alterne les phases d'inclusion et d'exclusion dans le parcours de l'héroïne, Chimamanda Ngozi Adichie semble avoir digéré le genre littéraire de la romance dont Americanah se rapproche par bien des égards avec la quête initiatique, la dimension amoureuse au centre (le couple séparé Ifemulu-Obinze) et la structure de réconciliation finale où les deux amants se retrouvent à Lagos. Pour autant, l'autrice n'a pas vraiment recours aux phénomènes extraordinaires ni aux possibilités fantastiques qu'aurait pu lui offrir la pensée magique africaine. En fait, Americanah et ses bons principes n'est pas loin du roman sentimental.

À la manière de Ségou de Maryse Condé, Ghana Must Go s'apparente au genre de la saga: le récit entremêle les souvenirs du patriarche mourant et les expériences de vie des autres membres de la famille pour raconter l'histoire des Sai, sur fond d'une planète mondialisée. Le vécu diasporique des quatre enfants Sai, leurs allers-retours 
intercontinentaux, participent d'une poétique du déplacement qui contribue à la construction d'une identité transnationale. Le titre original du roman, Ghana Must Go, évoque un épisode difficile des relations entre Ghana et Nigeria, l'expulsion de deux millions de Ghanéens par le gouvernement du Nigeria à l'hiver 1983 (Selasi 2014a: 237; Selasi 2014b: 277). La traduction retenue en allemand par l'éditeur Fischerverlag, Diese Dinge geschehen nicht einfach so ["Ces choses-là n'arrivent pas comme ça”], est métaphorique et renvoie à une forme de déterminisme impliquant des relations d'interdépendance entre les vies, les histoires et les lieux. Les éditions Gallimard ont, elles aussi, fait le choix d'effacer la référence historique liée à la nation ghanéenne, peu identifiable par le lecteur non africain, pour choisir un titre à la fois poétique et symbolique, Le Ravissement des Innocents. L'expression "ravissement des innocents" apparaît pour la première fois dans l'image de deux enfants misérables du Ghana, se tenant la main et souriant avec "une faculté instinctive de se moquer du monde tel qu'il est" (idem: 41), ("an instinct to laugh at the world as they found it" (Selasi 2014a: 25)): "il [Kweku] avait pris cela pour de la sottise, le ravissement des innocents" (Selasi 2014b: 42), ("he'd mistaken it for silliness, the blitheness of the youngest" (Selasi 2014a: 25)). Taiye Selasi précise dans une interview:

Oui, c'est aussi comme cela que j'ai pensé les enfants de Kweku, les jumeaux Taiwo et Kehinde, que j'ai imaginés un peu comme Wendy et Peter Pan, capables de se tenir hors du monde et en dehors de ses horreurs. Ils ont la faculté de retourner au temps de l'innocence. (Selasi apud Stélandre 2014: s.p.)

Mais le ravissement qui désigne une manière d'être enlevé à soi-même constitue également une annonce du massacre des innocents. Il est vrai que le roman se construit sur des silences, des non-dits comme les raisons du départ de Kweku de l'hôpital où il exerçait, mais aussi une catastrophe en suspens et tend vers une révélation qui se situe au cœur du roman: qu'est-il arrivé aux jumeaux Taiwo et Kehinde au Nigeria? L'on apprendra qu'ils ont été victimes d'abus sexuels de la part d'un oncle pédophile (Selasi 2014a: 286; Selasi 2014b: 332). Le roman développe une poétique de l'ellipse et de la discontinuité, mimétique d’une séparation quasi ontologique entre Amérique et Afrique, 
explorant les failles de nos vies, ces éloignements et départs qui nous construisent: des exils géographiques comme intimes, la frontière entre une existence projetée, rêvée et celle qui est notre quotidien, ces "distances d'un autre ordre et plus difficiles à appréhender" que sont "douleur, colère, chagrin calcifié et ces questions laissées en suspens ou sans réponse depuis trop longtemps, silence et honte entre des générations de père et de fils". (Marcandier 2016: s.p.)

La poétique de l'ellipse et de l'indirection de Taiye Selasi s'oppose à l'esthétique de la plénitude et de la saturation à l'œuvre dans l'écriture réaliste de Chimamanda Ngozi Adichie, qui remplit par une série concertée d'analepses tous les vides creusés par le récit de la vie d'Ifemelu et d'Obinze jusqu'à leurs retrouvailles à Lagos.

L'espace transatlantique, lieu de transferts et d'échanges multipolaires, se présente donc comme morphologie de configurations discursives dont la caractéristique est de travailler à la promotion d'une poétique transnationale, où la quête des origines s'efface au profit de l'exploration de l'imaginaire de tous les lieux vécus, parcourus par une dynamique constante de déterritorialisation et de reterritorialisation.

\section{Conclusion}

L'écriture des autrices transatlantiques que sont Maryse Condé, Taiye Selasi et Chimamanda Ngozi Adichie pratique une (re)configuration littéraire des identités féminines - et masculines - à travers l'espace atlantique. Elle exprime une critique des inégalités entre hommes et femmes, mais sans détestation revendiquée du mâle supposé dominant. Elles manifestent une aptitude à la déterritorialisation - et l'espace atlantique s'y prête - tout en affichant un intérêt pour les relations interpersonnelles et sociales au sein de l'histoire collective. L'engagement politique passe par une parole personnelle, établie dès leurs premières œuvres pour Adichie et Selasi, mais acquise par Condé qui passe du roman historique à la Ségou à un récit à la première personne dans Moi, Tituba Sorcière... Il ne s'agit plus de faire l'histoire du peuple noir, mais de s'intéresser à des mémoires individuelles tout en transcendant les clivages nationaux ou ethniques, attitude caractéristique de la "Black Atlantic" selon Paul Gilroy (2003: 19). 
Aucune des trois ne s'inscrit dans un féminisme radical, ce qui induit une créativité littéraire plus libre, que l'on peut parfois situer dans la perspective du "womanism" d'Alice Walker (1983), féminisme afro-américain, qui se distingue du féminisme occidental par son insistance sur la dimension culturelle et l'héritage matrilinéaire, même si aucune des trois ne s'en réclame. L'écriture de Maryse Condé, Taiye Selasi et Chimamanda Ngozi Adichie se fait sans esprit de système ou d'école et écartent a priori le carcan des théories. Dans le monde francophone, la négritude, l'antillanité ou la créolité ont parfois été présentées comme des théories de conception masculine à tendance universalisante qui ne laissent guère de place aux femmes (Chancé 2009). Et l'on sait ce que Wole Soyinka et les écrivains africains anglophones pensaient de la négritude et de sa possible dérive essentialisante. Mais on pourra aussi objecter que l'œuvre transatlantique de Chimamanda Ngozi Adichie et de Taiye Selasi répond au marketing des éditeurs occidentaux de manière à complaire au goût de l'Occident et à satisfaire son appétit de littératures marginales et de commodités exotiques (Huggan 2001), mais aussi de cultures cosmopolites raffinées et de sophistications interculturelles (Ponzanesi 2014: 4). La stratégie éditoriale peut conduire à élaborer une "image d'auteur" (Amossy 2009) à la fois construite sur des clichés de la féminité et obéissant à des postures postcoloniales complexes par rapport au champ littéraire et culturel (Mangeon 2012): d'une beauté sculpturale et toujours vêtue avec une grande distinction sans parler de sa splendide coiffure afro, Taiye Selasi est en passe de devenir une icône médiatique, une figure "virale" au même titre que Chimamanda Ngozi Adichie, leader d'opinion, très présente sur les réseaux sociaux.

Même si elles ont pu être écrites depuis les marges, les œuvres de Maryse Condé, Taiye Selasi et Chimamanda Ngozi Adichie font autorité et finissent par définir un horizon d'attente qui devient la norme et tendent à poser un nouveau canon. Si Ségou et Moi, Tituba Sorcière... peuvent passer désormais pour des "classiques" de la littérature francophone postcoloniale, les romans de Taiye Selasi et Chimamanda Ngozi Adichie s'inscrivent dans la world literature, où elles tiennent une place importante par le nombre des traductions, en plus d'une trentaine de langues pour chacun de leurs romans phares, ce qui leur confère une visibilité planétaire liée à la célébrité. Quant à l'œuvre de Maryse Condé, elle a été 
traduite dans de nombreuses langues, notamment en anglais par le mari de l'autrice, Richard Philcox, ce qui pose une collaboration auteur-traducteur très intime. Ces trois autrices transatlantiques sont réellement du monde au sens du concept de "worldliness" d'Edward Said (1983), qui implique engagement et mondanité, ce sont des écrivaines globales, des "autrices-monde" par leur appartenance à la "littérature-monde" et à la world literature.

\section{NOTES}

\footnotetext{
${ }^{1}$ Dans sa séance du jeudi 28 février 2019, l'Académie française a adopté à une large majorité le rapport sur la féminisation des noms de métiers et de fonctions présenté par une commission d'étude comprenant quatre de ses membres (<http://www.academie-francaise.fr/sites/academie-francaise.fr/files/rapport_feminisation noms_de_metier_et_de_fonction.pdf>).

${ }^{2}$ Gayatri Chakravorty Spivak a fini par récuser l'expression qui a, selon elle, entraîné trop de confusions, mais ne s'oppose pas à ce qu'elle soit utilisée par d'autres.

${ }^{3}$ L'usage, en français, est de parler de "la" Black Atlantic, sans doute en référence au nom "diaspora" qui est féminin.
} 


\section{Bibliographie}

Adichie, Chimamanda Ngozi (2018), Nous Sommes Toutes des Féministes, trad. Sylvie Schneiter, Paris, Gallimard, "Folio" [We Should All Be Feminists [2012]].

-- (2016) Americanah, trad. Anne Damour, Paris, Gallimard, "Folio" [2014].

-- (2014), Americanah, Londres, Fourth Estate [2013].

Akfbar, Arifa (2011), "Is Feminism Relevant to 21 1 st-Century Fiction?", The Independent, 13 mai, s.p., <https://www.independent.co.uk/arts-entertainment/books/features/isfeminism-relevant-to-21st-century-fiction-2283009.html> (dernière consultation le $2 / 02 / 2019)$.

Alix, Florian (2017), “L'Afropolitanisme au Féminin: Une Déterritorialisation des lieux communs?", Études Littéraires Africaines, n 44, 113-127.

Amossy, Ruth (2009), "La Double Nature de l'Image d'Auteur", Argumentation et Analyse du Discours, no 3, s.p., <http://journals.openedition.org/aad/662> (dernière consultation le 30/01/2019).

Bénichou, Paul (1973), Le Sacre de l'Écrivain, 1750-1780. Essai sur l'avènement d'un pouvoir spirituel laïque dans la France moderne, Paris, Joseph Corti.

Brugier, Julie (2016), “'Une Féministe! Qu'est-ce que c'est que cela?' Penser le féminisme et l'écriture féminine dans Moi, Tituba Sorcière... Noire de Salem (1986) et Célanire Cou-Coupé (2001) de Maryse Condé", SELF XX-XXI, Écriture Féminine aux xx et xxie Siècles, Entre Stéréotype et Concept, <https://self.hypotheses.org/publications-en-ligne/ecriturefeminine-aux-xxe-et-xxie-siecles-entre-stereotype-et-concept/rapports-ambivalents-alheritage-feministe-2> (dernière consultation le 26/01/2019).

Brunn, Alain / Martin François-René (2019), "Auteur", Encyclopædia Universalis, <http://www.universalis-edu.com/encyclopedie/auteur/> (dernière consultation le 
1/02/2019).

Chancé, Dominique (2009), "Maryse Condé, la Parole d'une Femme qui ne Serait pas la Femme", Horizons Maghrébins - Le Droit à la mémoire, $\mathrm{n}^{\circ}$ 60, 66-77.

Chanda, Tirthankar (2018), "Maryse Condé, monstre sacré des lettres francophones", <http://www.rfi.fr/ameriques/20181013-portrait-maryse-conde-nobel-alternatif-antillesfrancophonie-cesaire-fanon> (dernière consultation le 15/01/2019).

-- (2014), “Taiye Selasi: 'Je ne suis pas une femme littéraire normale”,, <http://www.rfi.fr/hebdo/20141031-taiye-selasi-litterature-ravissement-innocents> (dernière consultation le 10 décembre 2018).

Chaudet, Chloé (2017), “Des Trajectoires Féminines entre l’Afrique et les Amériques chez Chimamanda Ngozi Adichie, Maryse Condé, Waris Dirie et Toni Morrison: Vers une nouvelle pensée du féminisme et du roman transatlantique", in Histoire des Lettres Transatlantiques. Les Relations littéraires Afrique-Amériques, Bécherel, Les Perséides, "Le Monde Atlantique", 147-159.

Compagnon Antoine (s/d), “Qu'est-ce qu'un Auteur?", Fabula, <http://www.fabula.org/compagnon/auteur.php> (dernière consultation le 9/02/2019).

Condé, Maryse (2000), “La Race n'est pas Primordiale”, entretien avec Elizabeth Nuñez, Courrier de l'Unesco, 47-51.

-- (1986), Moi, Tituba Sorcière... Noire de Salem, Paris, Gallimard, "Folio".

-- (1985), Ségou. La Terre en miettes, Paris, Robert Laffont.

-- (1984), Ségou. Les Murailles de la terre, Paris, Robert Laffont.

Crenshaw, Kimberlé W. (1989), "Demarginalizing the Intersection of Race and Sex: A Black feminist critique of antidiscrimination doctrine, feminist theory and antiracist politics", University of Chicago Legal Forum, vol. 1989, issue 1, 139-167.

Dorlin, Elsa (2008), Black Feminism. Anthologie du féminisme africain-américain, 1975-2000, 
Paris, L'Harmattan.

Foucault, Michel (1969a), L’Archéologie du Savoir, Paris, Gallimard, “Nrf”.

-- (1969b), “Qu'est-ce qu'un Auteur?”, Bulletin de la Société Française de Philosophie, 63e année, $n^{\circ} 3,73-104$.

Gilroy, Paul (2003), L'Atlantique Noir. Modernité et double conscience, trad. Jean-Philippe Henquel, Paris, Kargo [The Black Atlantic. Modernity and double consciousness, Londres, Verso [1993]].

Gleeson, Sinead (2013), “Taiye Selasi: 'The Images that I see don't represent the Africa that I know"', The Irish Times, 11 mai, <https://www.irishtimes.com/culture/books/ taiye-selasithe-images-that-i-see-don-t-represent-the-africa-that-i-know-1.1388689> (dernière consultation le 11/01/2019).

Holst-Petersen, Kirsten / Rutherford, Anna (dir.) (1985), A Double Colonization: Colonial and post-colonial women's writing, Aarhus, Dangaroo.

hooks, bell (1986), "Sororité: la solidarité politique entre les femmes” ["Sisterhood: Political solidarity between Women", trad. Anne Robatel, Feminist Review, $\mathrm{n}^{\circ}$ 23, 1-24], $<$ https://infokiosques.net/lire.php?id_article=1161> (dernière consultation le 25/01/2019).

Huggan, Graham (2001), The Post-Colonial Exotic: Marketing the margins, London \& New York, Routledge.

Mangeon, Anthony (dir.) (2012), Postures Postcoloniales. Domaines africains et antillais, Paris, Karthala, "Lettres du Sud".

Marcandier, Christine (2016), “Taiye Selasi, Poésie de l'Exil Intérieur et Ravissement des Innocents", Diacriktik, 8 juin, <https://diacritik.com/2016/06/08/taiye-selasi-poesie-delexil-interieur-et-ravissement-des-innocents> (dernière consultation le 2/01/2019).

Miano, Léonora (2012), Habiter la Frontière, Paris, L’Arche. 
Pfaff, Françoise (1993), Entretiens avec Maryse Condé, Paris, Karthala.

Ponzanesi, Sandra (2014), The Postcolonial Cultural Industry. Icons, markets, mythologies, New York, Palgrave Macmillan.

Said, Edward (1983), The World, the Text and the Critic, Cambridge (Mass.), Harvard University Press.

Selasi, Taiye (2014a), Ghana Must Go, Londres, Penguin Books [2013].

-- (2014b), Le Ravissement des Innocents, trad. Sylvie Schneiter, Paris, Gallimard, “Du Monde Entier".

-- (2011), "The Sex Lives of African Girls", The F-Word, Granta, no. 115, $<$ https://granta.com/the-sex-lives-of-african-girls/> (dernière consultation le 12/02/2019).

Simon, Catherine (2015), “Chimamanda Ngozi Adichie, Impériale”, Le Monde des Livres, 5/02, <https://www.lemonde.fr/livres/article/2015/02/05/chimamanda-ngozi-adichieimperiale_4570126_3260.html> (dernière consultation le 10/02/2019).

Spivak, Gayatri Chakravorty (2011), Nationalisme et imagination, trad. Françoise Bouillot, Paris, Payot [Nationalism and the Imagination [2007]].

-- (2009a), En d'Autres Mondes, en d'Autres Mots: Essais de politique culturelle, trad. Françoise Bouillot, Paris, Payot [In Other Worlds: Essays in cultural politics [1987]] .

-- (2009b), Les Subalternes Peuvent-Elles Parler?, trad. Jérôme Vidal, Paris, Éditions Amsterdam [Can the Subaltern Speak? [1988]].

Stélandre, Thomas (2014), "Taiye Selasi: 'Je voulais que tous les protagonistes trouvent une maison dans l'amour'”, Libération, <http://www.liberation.fr/livres/2014/10/22/jevoulais-que-tous-les-protagonistes-trouvent-une-maison-dans-l-amour_1127259> (dernière consultation le 20/01/2019).

Viala, Alain (1985), Naissance de l'Écrivain. Sociologie de la littérature à l'âge classique, Paris, 
Minuit, "Le Sens Commun".

Viennot, Éliane (2017), Non, le Masculin ne l'Emporte pas sur le Féminin! Petite histoire des résistances de la langue française, nouvelle édition augmentée, Donnemarie-Dontilly, Éditions iXe [2014].

Walker, Alice (1983), In Search of our Mothers' Gardens: Womanist prose, San Diego, New York et Londres, Harcourt Brace Jovanovitch.

Yves Clavaron est professeur de littérature générale et comparée à l'Université Jean Monnet de Saint-Étienne. Ses travaux portent sur le postcolonialisme, la francophonie, l'écocritique dans les domaines anglophone et francophone. Il est l'auteur de Poétique $d u$ Roman Postcolonial (Publications Université de Saint-Étienne, 2011), Edward Said: L'Intifada de la culture (Kimé, 2013), Petite Introduction aux Postcolonial Studies (Kimé, 2015) et Francophonie, Postcolonialisme et Mondialisation (Classiques Garnier, 2018). Son ouvrage, Le Génie de l'Italie (Connaissances et Savoirs, 2006) a été réédité en 2017. Il a dirigé un ouvrage collectif avec Jean-Marc Moura, Histoire des Lettres Transatlantiques: Les Relations littéraires Afrique-Amériques (Bécherel, Les Perséides, 2017). 